\title{
Matematech: Plataforma de Apoio à Aprendizagem de Matemática nos Anos Iniciais do Ensino Fundamental
}

\author{
João Paulo P. de Araújo ${ }^{1,2}$, Gabriella C. Barbosa Costa ${ }^{2}$, José Geraldo R. Júnior ${ }^{2}$ \\ ${ }^{1}$ Departamento de Informática - Universidade Federal de Viçosa (UFV - MG) \\ Campus Universitário - s/n - Viçosa - MG - Brasil \\ ${ }^{2}$ Centro Federal de Educação Tecnológica de Minas Gerais (CEFET - MG) \\ Rua José Peres 558 - Centro - Leopoldina - MG - Brasil \\ joao.araujo2@ufv.br, \{gabriella,jgrjunior\}@leopoldina.cefetmg.br
}

\begin{abstract}
Due to challenges proposed by nowaday scenary in Brazilian education and considering the importance of technology to learning acquision, this article presents Matematech Platform: a technology on order to help teaching basic mathematic knowledge such as directions, space and arithmetic operations in early years of basic education. The Platform was developed used electronic device which are low cost and easy access. The Platform allows to introduce basic math concepts through ludic activities. Matematech Platform was applied in a group of students in a government school and it was noticed that the Platform is an efficient tool to insure a contextualized learning. This work was partially funded by FAPEMIG.
\end{abstract}

Resumo. Partindo dos desafios propostos pelo atual cenário em que se encontra a educação brasileira e tendo como princípio a importância da tecnologia para o aprendizado, este artigo apresenta a plataforma Matematech, uma solução tecnológica para auxiliar no ensino de conhecimentos básicos de matemática como direção, espaço e operações aritméticas, nos anos iniciais do Ensino Fundamental. A plataforma foi desenvolvida utilizando dispositivos eletrônicos de baixo custo e fácil acesso. Por meio dela, são trabalhados conceitos básicos de matemática através da ação lúdica. A plataforma Matematech foi aplicada a alunos de uma escola da rede pública e observou-se que ela é uma ferramenta eficiente na garantia de um aprendizado contextualizado. Este trabalho recebeu o apoio da FAPEMIG

\section{Introdução}

Na sociedade do século XXI, a tecnologia se faz presente em diversos setores e ela se encontra inserida na organização das mais variadas práticas sociais [RIBEIRO, 2012]. Atualmente, é impossível deixar de usar as novas tecnologias para a obtenção de conhecimento, uma vez que, nesta sociedade tecnológica da informação e da comunicação, o acesso à informação, o uso e a análise de dados e as tomadas de decisão fundamentadas em situações de incerteza faz em parte das novas necessidades de formação dos cidadãos [VALLECILLOS e MORENO, 2002].

Além da necessidade citada anteriormente, a educação brasileira tem vivenciado um momento delicado. Apesar de ser pauta principal das políticas públicas contemporâneas, os resultados alcançados não se apresentam satisfatórios. Tomando como 
V Congresso Brasileiro de Informática na Educação (CBIE 2016)

Anais do XXVII Simpósio Brasileiro de Informática na Educação (SBIE 2016)

referência avaliações nacionais e internacionais, que medem o nível de conhecimento dos jovens da educação básica, como, por exemplo, o PISA (Programme for International Student Assessment) e a Prova Brasil, o Brasil está longe de garantir uma educação pública de qualidade. De acordo com os dados do PISA [AVVISATI, 2012], em 2012, o Brasil caiu quatro posições em relação a 2009 , ocupando a $58^{a}$ posição, numa escala de 65 países. Segundo relatório divulgado pela OCDE (Organização para a Cooperação e Desenvolvimento Econômico), na disciplina de matemática, apesar da modesta melhora, o país caiu da $57^{\text {a }}$ posição para a $58^{\text {a }}$ [AVVISATI, 2012]. Segundo os resultados da edição de 2013 da Prova Brasil [QEDU, 2013], somente 40\% dos alunos aprenderam o adequado na competência de leitura e interpretação de textos até o $5^{\circ}$ ano na rede pública de ensino. $\mathrm{Na}$ disciplina de matemática, o resultado é ainda mais crítico. Apenas $35 \%$ dos alunos aprenderam o adequado na competência de resolução de problemas até o $5^{\circ}$ ano na rede pública de ensino [QEDU, 2013].

Diante do cenário apresentado, é preciso entender a razão que leva a maioria dos alunos a não conseguir aprender matemática. Está evidente que algo precisa ser feito para mudar essa realidade, uma vez que a metodologia atual não está sendo suficiente, pelo menos para a maioria absoluta dos alunos que se encontram no $5^{\circ}$ ano do Ensino Fundamental. Um dos caminhos para essa mudança está no desenvolvimento de soluções tecnológicas para auxiliar no aprendizado. Na área educacional, as tecnologias, se bem utilizadas, devem contribuir para auxiliar no processo de aprendizagem e para disseminar o acesso de todos à educação básica [NETO et al., 2013]. Sabe-se que, por si só, as tecnologias não resolverão todos os problemas da educação brasileira, que também envolve formação de professores, participação familiar, infraestrutura, entre outros. No entanto, são inúmeros os casos em que as Tecnologias Digitais da Informação e Comunicação (TDIC) proporcionam melhorias na forma como o conhecimento é construído, auxiliando professores, pais e alunos na busca por um aprendizado mais contextualizado [TODA et al., 2014]. Um exemplo de aplicação de TDIC está na utilização de jogos tecnológicos como ferramenta no auxílio ao aprendizado, que pode levar à criação de situações de ensino e aprendizagem motivadoras, dinâmicas e envolventes [CARVALHO et al., 2015].

Tendo como base as questões discutidas, este trabalho tem como objetivo a apresentação de uma plataforma tecnológica de baixo custo, acessível às escolas, e que auxilie no processo de alfabetização matemática nos anos iniciais do Ensino Fundamental. A plataforma, chamada Matematech, é composta por dispositivos eletrônicos de baixo custo e fácil acesso. Através dela, são trabalhados, por meio da ação lúdica e da contextualização à realidade vivenciada, conceitos básicos, como direção, espaço e operações aritméticas, que são essenciais para o desenvolvimento do pensamento lógico matemático. Experimentos realizados em escolas demonstram que a plataforma contribui no processo de aprendizagem, melhorando o nível de absorção do conhecimento por parte dos alunos.

O restante do artigo apresenta-se da seguinte maneira: na Seção 2, é realizada uma análise de algumas propostas de projetos tecnológicos que auxiliam no aprendizado de matemática; a Seção 3 descreve sobre a metodologia utilizada; a seção 4 traz o processo de construção da plataforma Matematech, demonstrando a lógica, métodos e dispositivos utilizados; a Seção 5 detalha os experimentos realizados e analisa os resultados 
V Congresso Brasileiro de Informática na Educação (CBIE 2016)

Anais do XXVII Simpósio Brasileiro de Informática na Educação (SBIE 2016)

alcançados, e, por fim, a Seção 6 traz as conclusões do trabalho.

\section{Trabalhos Relacionados}

No contexto mundial, tem-se a Khan Academy [TAVARES et al., 2012], uma plataforma on-line que fornece videoaulas e atividades que podem ser acessadas de forma gratuita pelo usuário. Uma vantagem dessa plataforma é possibilitar tanto um ensino personalizado quanto o acompanhamento por parte do professor a respeito do desempenho dos alunos.

Em território nacional, existem vários projetos com o objetivo de ajudar no aprendizado de matemática por meio da tecnologia. Entre eles, é possível destacar o FORMEL [GONÇALVES, 2004], que consiste em um ambiente computacional favorável à aprendizagem da matemática por meio de um jogo educativo. No jogo, o objetivo é conduzir a formiga até o mel, fazendo uso apenas dos movimentos do teclado: para cima, para baixo, para a esquerda e para a direita. A situação vivenciada possibilita que a criança proponha a construção de figuras geométricas, tais como quadrados e retângulos. Baseado nos passos da formiga, pode-se contar e verificar suas medidas por meio dos pixels (pontos na tela). Nesse jogo, também são trabalhadas as questões de caminho mínimo e máximo.

Em ambos os projetos analisados, o uso da Internet é um pré-requisito fundamental para a aplicação das ferramentas. Levando em consideração que a atual estrutura das escolas, em termos de conexão de rede, não atende a essa necessidade, a plataforma Matematech apresenta como vantagem a não utilização da Internet para o desenvolvimento das atividades. Além disso, as propostas citadas apresentam um custo elevado para a implantação dentro do ambiente escolar, por serem dependentes de computadores ou dispositivos móveis, como tablets ou smartphones. Na construção da plataforma Matematech, a utilização de dispositivos eletrônicos de baixo custo foi uma das prioridades, visando tornar a tecnologia desenvolvida mais acessível à realidade da escola pública. Outra vantagem da plataforma Matematech está na facilidade de uso, não exigindo um conhecimento tecnológico prévio por parte dos professores.

\section{Metodologia}

Com base nos baixos resultados dos alunos na disciplina de matemática em avaliações como o PISA e a Prova Brasil, idealizou-se o desenvolvimento da plataforma Matematech, que auxilia no aprendizado de conceitos básicos da matemática de crianças que se encontram nos anos iniciais do ensino fundamental. A metodologia utilizada na concepção da plataforma foi comporta de 12 etapas: (1) Identificação e estudo do problema; (2) Organização da solução; (3) Definição do escopo da plataforma; (4) Desenvolvimento do software da plataforma; (5) Montagem do hardware da plataforma; (6) Junção entre o software e o hardware; (7) Testes da plataforma; (8) Elaboração e aplicação da avaliação diagnóstica na escola; (9) Aplicação da plataforma na escola; (10) Aplicação da avaliação diagnóstica; (11) Análise dos resultados; (12) Organização e publicação dos resultados.

\section{Plataforma Matematech}

A plataforma Matematech (Figura 1) tem como objetivo colaborar no ensino aprendizagem de matemática dos alunos dos anos iniciais da educação básica. O processo 
V Congresso Brasileiro de Informática na Educação (CBIE 2016)

Anais do XXVII Simpósio Brasileiro de Informática na Educação (SBIE 2016)

de construção da plataforma foi pautado nas possibilidades e limitações das escolas, dos professores e dos próprios alunos em relação ao uso de tecnologias. Dessa forma, foi construído um instrumento didático e acessível à realidade das escolas públicas. Assim, a plataforma foi construída tendo como base três características: (1) utiliza componentes de baixo custo, (2) não depende de recursos de informática, como computadores e acesso à Internet e (3) não exige do professor um avançado conhecimento tecnológico.

Por meio de situações contextualizadas e divertidas, o jogo proposto pela plataforma trabalha conceitos fundamentais da matemática e temas importantes contidos na matriz de referência do PROEB e PROVA BRASIL [INEP, 1998], como por exemplo, direção, espaço e operações aritméticas.
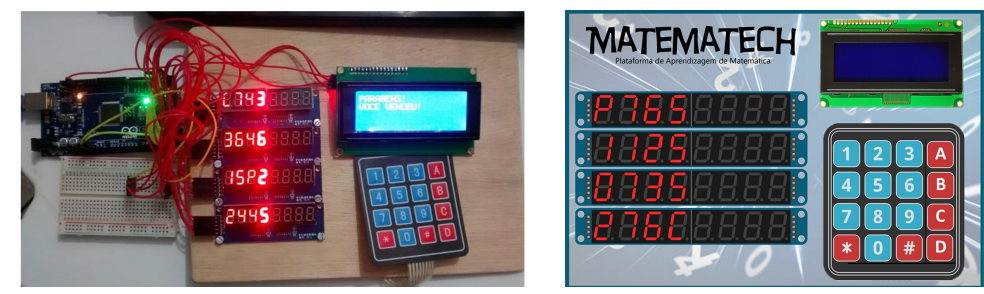

Figura 1. Protótipo da plataforma Matematech ao lado da projeção do produto finalizado.

\subsection{Funcionamento da Plataforma}

A versão atual do protótipo da plataforma Matematech apresenta dois níveis de dificuldade. Em cada nível, existem três fases que permitem ao aluno desenvolver conhecimentos importantes relacionados à direção, espaço e operações aritméticas. No jogo, o aluno deve sair de um ponto inicial e chegar até um ponto final, utilizando o menor número de passos possível, indicando o caminho que ele percorreu para chegar no ponto final, utilizando as direções. Por último, ele deve indicar a soma dos valores contidos no caminho percorrido por ele.

Ao iniciar o jogo, o aluno tem uma opção de "MENU" para escolha do nível que ele deseja jogar: "1- Nível 1" ou "2- Nível 2". Para cada nível, existem 3 fases. No Nível 1, é gerada uma matriz quadrada de ordem 3 e, no Nível 2, uma matriz quadrada de ordem 4. O nível de dificuldade pode variar tanto pelo tamanho da matriz quanto pela grandeza dos números utilizados na matriz. Após a escolha do nível, o programa define onde estarão os pontos "P" e "C", que significam, respectivamente, Partida e Chegada. Para essa definição, é utilizado um sorteio, levando em consideração a quantidade de linhas e colunas da matriz, buscando manter o ponto "P" distante do ponto "C", de forma a permitir que o aluno, em algum momento, tenha que modificar o sentido que esteja percorrendo, e trabalhe conceitos de "direita e esquerda".

Após a definição e exibição dos pontos de partida e de chegada, a variável de controle habilita o início da Fase 1 do programa, que consiste na definição do menor caminho a ser percorrido entre a Partida e a Chegada. Se o aluno acertar, passará para a segunda fase do jogo. Caso erre, continuará tentando, até que consiga avançar de fase.

Para acompanhar o processo de evolução dos alunos e incentivá-los a superar suas limitações, definiu-se um sistema de pontuação. A cada fase que o aluno avança, ele acumula pontos, podendo chegar ao valor máximo de 50 pontos. Em cada fase, o aluno 
V Congresso Brasileiro de Informática na Educação (CBIE 2016)

Anais do XXVII Simpósio Brasileiro de Informática na Educação (SBIE 2016)

tem a possibilidade de adquirir 10 pontos; porém, a Fase 1 e a Fase 2 possuem peso 2, devido ao grau de dificuldade. Caso o aluno tenha um erro, esse erro não é trabalhado de forma a puni-lo, mas é visto apenas como um indicador de que é necessário aprimorar determinadas habilidades. Sendo assim, em cada erro, o aluno perde apenas 1 ponto na fase 1 e 2 pontos nas fases 2 e 3 , de forma a diferenciar o tipo de erro em cada etapa. Ao final, mesmo que o aluno tenha, por exemplo, dez erros na fase 1, ele ainda acumula um total de 40 pontos. Esse resultado permite ao professor constatar que o aluno precisa estudar mais e, ao mesmo tempo, não o deixa desmotivado.

Na Fase 2, o aluno define o sentido e a direção do caminho indicado por ele (na fase 1) como o menor para sair de "P" e chegar até " $C$ ". $O$ aluno entra com a quantidade de sentidos (D- Direita, E- Esquerda, C-Cima e B-Baixo) que serão armazenados em um único vetor, que mais tarde será percorrido para verificar a veracidade das informações fornecidas. Caso os dados não resultem na chegada até o ponto "C", o programa informa o erro e solicita que ele digite novamente até que o mesmo acerte o valor. Caso o aluno acerte, a variável de controle habilita o início da Fase 3.

Na Fase 3, o aluno soma os valores contidos nas casas que ele percorreu para sair de "P" e chegar até " $C$ ". Após o aluno informar esse valor, ele calcula se o mesmo é igual ao valor da soma dos valores das casas percorridas. Se for idêntico, o display LCD exibe uma mensagem indicando que o aluno venceu esse nível e será mostrada a pontuação conquistada. Caso contrário, será solicitado que ele envie novamente um valor.

\subsubsection{Fase 1: Determinação do Menor Caminho}

Na Fase 1, o aluno precisa indicar qual o número de casas, com base no menor caminho que ele precisa que percorrer para sair de "P"(Partida) e alcançar "C"(Chegada). Ao praticar essa fase, são trabalhadas questões envolvendo espaço, grandezas e medidas, números e operações, além do tratamento de informações.

O algoritmo Dijkstra [CARVALHO, 2005] foi utilizado para solucionar o problema de identificação do menor caminho. Determinado um vértice como raiz da busca, esse algoritmo calcula o custo mínimo do vértice para todos os outros vértices do grafo. Dijkstra utiliza uma lógica bastante simples e possui um alto nível de desempenho. No entanto, ele não garante a precisão da solução no caso de existir a presença de arcos com valores negativos, que não é o caso da Plataforma apresentada. Nesse projeto, o algoritmo é usado sobre grafos não orientados [CARVALHO, 2005]. Porém, antes de aplicar esse algoritmo, é necessária a construção de uma matriz de adjacência. A matriz de adjacência de um determinado grafo possui linhas e colunas indexadas pelos vértices. Se $B$ for a matriz de adjacências de índices $i$ e $j, B(i, j)=1$ se os vértices $i$ e $j$ estiverem conectados por uma aresta e $B(i, j)=0$ caso a situação seja oposta. Existem dois tipos de grafos: os direcionados e os não direcionados. Observa-se que em grafos não direcionados, a matriz de adjacências é sempre simétrica ao longo da diagonal principal, ou seja, $B(i, j)=B(j, i)$. No caso desse algoritmo, o grafo é não direcionado, simples e possui peso nas arestas (1 ou 999).

A versão atual da plataforma, por limitações de hardware, possui 2 níveis, mas pode assumir mais níveis em função da flexibilidade com que as variáveis foram defini- 
V Congresso Brasileiro de Informática na Educação (CBIE 2016)

Anais do XXVII Simpósio Brasileiro de Informática na Educação (SBIE 2016)

das. No Nível 1, é formada uma matriz $3 \times 3$ e, no nível 2, uma matriz 4x4. Em função disso, teremos matrizes de adjacência diferentes para cada situação. Para atender a essas necessidades do projeto, foram feitas algumas adaptações lógicas para o preenchimento da matriz de adjacência, forçando alguns resultados, como a atribuição às arestas conectas em diagonal o valor 0 , indicando que esse caminho não existe, devido ao fato de o jogo proposto pela plataforma não percorrer caminhos na diagonal.

A partir da análise das matrizes de adjacência, fez-se necessário construir uma lógica para o preenchimento de forma automática, independente do nível escolhido pelo usuário. Analisando as matrizes, foi identificado, em um primeiro momento, que todos os pontos em que a diferença entre vértices é igual a 1 ou a $N$, em que $N$ é o valor da ordem da matriz, existe conectividade entre os vértices, e, dessa forma, eles recebem o valor 1. Onde essa regra não se aplica, os vértices não se conectavam e é atribuído valor 0 . Porém, percebeu-se que existe uma exceção para essa regra, uma vez que, nas laterais de ambos os grafos, essa regra não se aplica. Por exemplo, para o Nível 1, entre os vértices 3 e 4, a diferença é 1 e, no caso, não há aresta que os conecte diretamente, ou seja, ele deve receber valor 0 , mas está recebendo valor 1 . O mesmo acontece para o Nível 2, em que, por exemplo, os vértices 8 e 9 também apresentam diferença igual a 1, mas não apresentam conectividade entre eles. Essas exceções foram tratadas. Depois que a matriz de adjacência está formada, é executado o algoritmo de Dijkstra.

\subsubsection{Fase 2: Sentido e Direção do Menor Caminho}

Ao atingir a Fase 2, o aluno deve indicar o sentido e a direção do menor caminho que ele percorreu na Fase 1. Nessa fase, o aluno consolida conceitos relacionados a espaço, em que se trabalha a localização/movimentação de objeto em representações gráficas, além do tratamento de informações.

Quando o aluno digitar o sentido "D", o algoritmo soma o valor 1 à posição local na coluna. Quando o aluno digitar o sentido "E", o algoritmo subtrai o valor 1 da variável que indica a coluna onde está localizado. Quando o aluno digitar o sentido "C", o algoritmo subtrai o valor 1 da variável que indica a linha, enquanto ao digitar o sentido "B", o algoritmo soma o valor 1 à variável que indica a linha onde está localizado. Neste momento, o programa recebe os valores de entrada e armazena em um vetor do tamanho do menor caminho indicado pelo aluno na Fase 1. Mais adiante, uma função irá analisar um elemento de cada vez desse vetor. A cada vetor analisado, o programa verifica o limite de movimentação. Por exemplo, no caso de "D" e "B", uma variável de controle armazena o passo que o usuário deu e verifica se ele ultrapassará o limite da ordem da matriz. O mesmo acontece para as direções "C" e "B", uma vez que a variável que armazena o passo que o usuário avançou não pode ser inferior a 0 . Assim sendo, ao final da inserção de dados, se a posição final for igual à posição em que se encontra o Ponto C, ele acertou e avança de fase; caso contrário, a criança deverá digitar novamente as posições que precisa percorrer para sair de "P"e chegar até "C". 
V Congresso Brasileiro de Informática na Educação (CBIE 2016)

Anais do XXVII Simpósio Brasileiro de Informática na Educação (SBIE 2016)

\subsubsection{Fase 3: Soma dos Valores do Caminho Percorrido}

A última fase do programa solicita ao usuário que ele insira a soma dos valores contidos nas casas que ele percorreu para sair de "P"e chegar até "C". Nessa fase, o aluno tem a oportunidade de praticar conhecimentos em torno de números e operações. Ele deve calcular o resultado de uma adição e resolver problemas com números naturais, envolvendo diferentes significados da adição: juntar, alteração de um estado inicial, comparação e mais de uma transformação. É possível também construir o conhecimento por meio do tratamento de informações, em que o aluno lê as informações e os dados apresentados.

Após o aluno digitar o valor da soma, o programa irá analisar se esse valor é igual ao valor da soma dos números das casas percorridas durante a Fase 1. Caso o valor esteja correto, o aluno venceu essa etapa, e consequentemente, finalizou o jogo. Caso contrário, será solicitado que o aluno entre com o valor correto da soma. Ao finalizar o jogo, é exibida uma mensagem de "Parabéns" e são exibidos os pontos adquiridos.

\subsection{Montagem Física da Plataforma}

A montagem física da plataforma é bastante simples, o que possibilita àqueles que tenham o software em mãos replicar o protótipo. Nela, utilizou-se, por questões de necessidade de memória, um Arduino Mega 2560, 4 módulos de 8 dígitos de display de 7 segmentos, 1 display LCD e 1 teclado matricial $4 \times 4$ (Figura 1). Todos os dispositivos são de baixo custo e fáceis de serem adquiridos e manipulados.

Para a exibição da matriz, que é a base para o jogo proposto pela Matematech, utilizaram-se 4 módulos contendo 8 dígitos de display de 7 segmentos cada um. Para utilizar esse módulo, é preciso conectar, além dos dois pinos de alimentação, três pinos ao Arduino (DIO, SCK e RCK). Esse módulo é uma alternativa para quem não quer utilizar uma série de fios para conectar display de 7 segmentos à placa Arduino. Para exibir os valores gerados da matriz nesses módulos, utilizou-se a biblioteca D3461BS.h, desenvolvida por alunos do curso técnico de Informática CEFET/MG, unidade Leopoldina. O uso da biblioteca D3461BS tem como objetivo simplificar o uso do display 3461BS, que possui 8 dígitos de 7 segmentos, no Arduino.

Levando em consideração que a Plataforma desenvolvida é um jogo e exige interação com o usuário, é utilizado um display de LCD para exibir as mensagens. No protótipo desenvolvido, foi utilizado um display LCD I2C 20x4. A vantagem de utilizar um display LCD I2C é que, com esse módulo, consegue-se fazer o controle do dispositivo LCD, seja ele $16 \times 2$ ou 20x4, fazendo uso, além dos pinos de alimentação, de apenas de dois pinos analógicos (SDA e SCL) da placa Arduino. Qualquer outro tipo de display LCD utilizaria no mínimo 6 portas para conexão, e em situações que se utiliza o Arduino UNO, sacrificar esse número de portas pode comprometer a finalização do protótipo.

\section{Experimentos e Análise dos Resultados}

Para mensurar a eficiência da plataforma Matematech, direcionaram-se os trabalhos para a aplicabilidade da mesma dentro do ambiente escolar. Esse experimento foi desenvolvido durante os meses de março e abril de 2016 com 15 alunos das turmas de $4^{\circ}$ e $5^{\circ}$ ano do Ensino Fundamental de uma escola pertencente à rede estadual de ensino no município de Leopoldina, Minas Gerais. 
V Congresso Brasileiro de Informática na Educação (CBIE 2016)

Anais do XXVII Simpósio Brasileiro de Informática na Educação (SBIE 2016)

A primeira ação desenvolvida com os alunos foi a realização de uma atividade diagnóstica. A partir dessa atividade, foi possível averiguar os conhecimentos que os discentes possuíam a respeito dos itens avaliados pela plataforma, assim como também, utilizando essa atividade inicial, foi possível comparar, ao final de todo o experimento, os avanços dos discentes com a utilização da plataforma Matematech.

As questões da atividade diagnóstica foram retiradas e adaptadas do PDE/PROVA BRASIL, dos anos de 2011 e 2013 [EDUCAÇÃO, 2007]. As questões propostas trabalham itens da matriz de referência da PROVA BRASIL como por exemplo, espaço e forma, medidas, números e operações e tratamento da informação [INEP, 1998]. Essas são questões que exemplificam os objetivos que norteiam a criação da Plataforma.

A realização dessa atividade apontou alguns resultados. O primeiro é que os alunos não mostraram entusiasmo ao desenvolver a atividade teórica. Em relação ao nível de conhecimento, muitos alunos não dominavam o conhecimento teórico, e os que sabiam as operações básicas, não conseguiam aplicá-las aos problemas apresentados. De acordo com as respostas dadas pelos alunos, pode-se afirmar que a maioria não domina $o$ conhecimento relativo ao reconhecimento da posição de um objeto em referência a outro, ou seja, não conseguem diferenciar direita de esquerda. O gráfico da Figura 2 mostra o número de alunos que acertou cada questão.

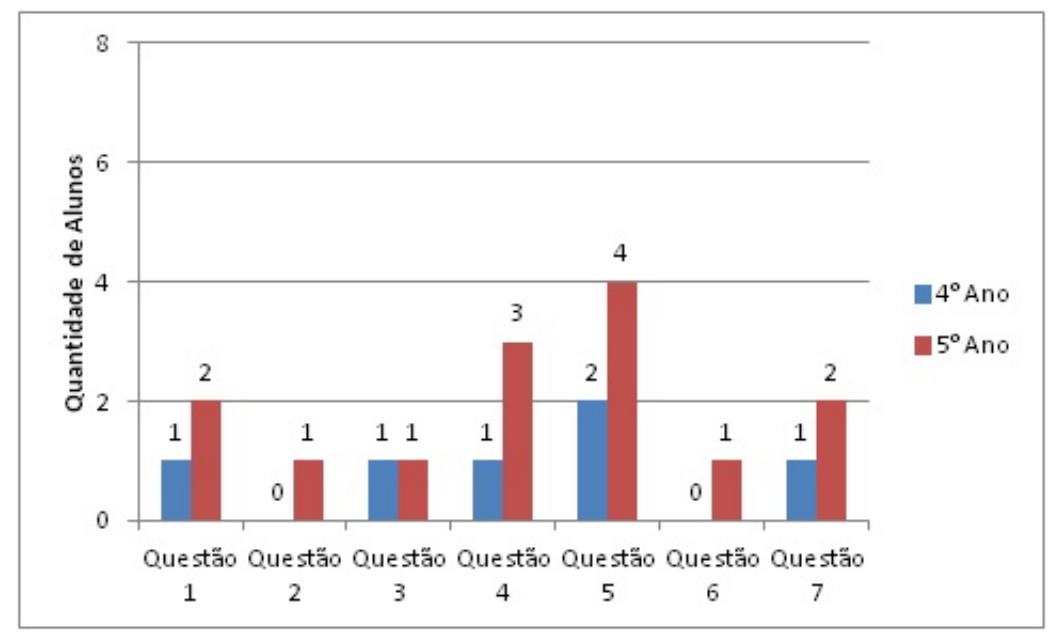

Figura 2. Quantidade de alunos que acertou cada questão.

Depois da atividade diagnóstica, foi iniciado o trabalho prático com a plataforma Matematech. Uma vez por semana, durante cinco semanas, cada aluno teve a oportunidade de brincar e aprender com a Plataforma.

A partir das observações realizadas, é possível fazer alguns apontamentos. Em relação aos 7 alunos do $4^{\circ}$ ano, no início, todos tiveram dificuldades em avançar nas fases do Nível 1. No terceiro dia de teste com a Plataforma, 5 alunos conseguiram vencer o Nível 1, apresentando resultados melhores se comparados com as pontuações anteriores. No final dos testes, 2 alunos não conseguiram completar essa fase. Com relação aos 5 alunos que venceram o Nível 1, estes praticaram o Nível 2 nas sessões que se seguiram e encontraram dificuldades para finalizar essa etapa, que foi concluída, após várias tentativas, por apenas 1 aluno no último dia de teste. 
V Congresso Brasileiro de Informática na Educação (CBIE 2016)

Anais do XXVII Simpósio Brasileiro de Informática na Educação (SBIE 2016)

Em relação aos 8 alunos do $5^{\circ}$ Ano, 4 alunos conseguiram avançar para o Nível 2 logo no segundo dia do teste, a outra metade teve que praticar mais algumas vezes para alcançar o Nível 2. Nesse nível, apenas 2 alunos tiveram dificuldades para finalizar. Mas no final dos cinco dias de teste, todos conseguiram chegar até o final.

Após a fase de testes na Plataforma, foi aplicada novamente a atividade diagnóstica. O objetivo, nesse momento, era obter dados para comparar com a primeira atividade aplicada e verificar se houve avanço nos conhecimentos trabalhados pela plataforma. O gráfico da Figura 3 mostra a quantidade de alunos que acertaram cada questão após o uso da plataforma, em que fica evidente a evolução de cada um.

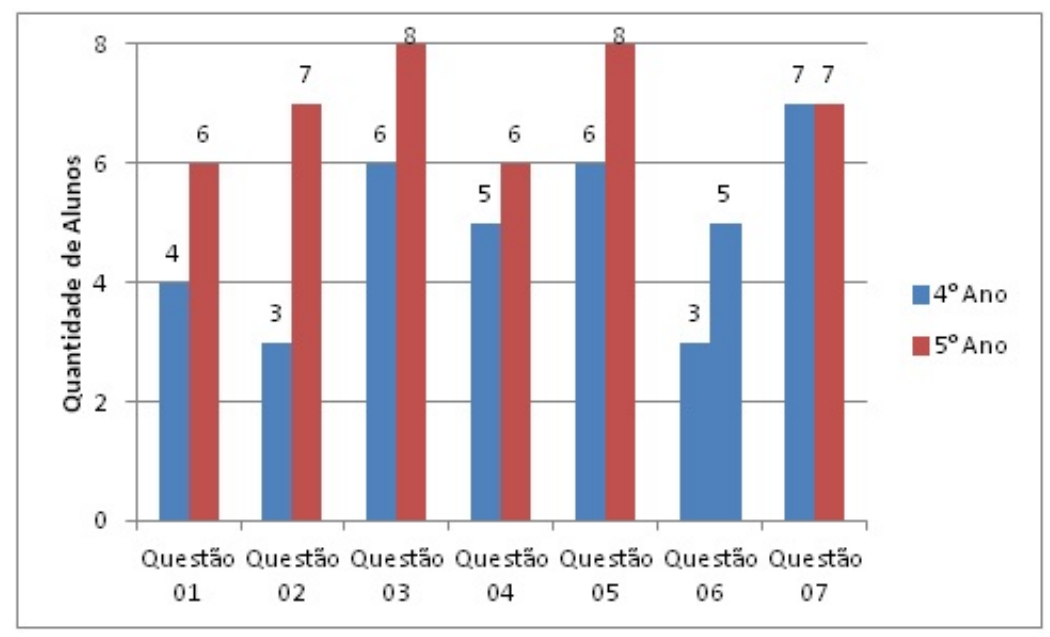

Figura 3. Quantidade de alunos que acertou cada questão.

Com a finalização desse processo, foi possível perceber que a plataforma proposta é bastante eficiente no auxílio à aprendizagem de matemática. O comportamento dos alunos deixou claro o interesse de cada um em participar da atividade. As próprias professoras que acompanharam o processo relataram que a plataforma despertou o interesse até dos alunos que pouco se interessavam por matemática.

\section{Conclusão}

Esse artigo apresentou a plataforma Matematech. Por meio dela, são trabalhados conceitos básicos de matemática por meio de ação lúdica. A plataforma Matematech foi aplicada a alunos dos anos iniciais do Ensino Fundamental de uma escola da rede pública, onde foi possível observar sua eficiência na garantia de um aprendizado contextualizado.

Tomando como base os elementos que nortearam o início desse projeto, pode-se afirmar que as metas projetadas foram alcançadas. Construi-se uma plataforma que fosse acessível às escolas. A construção da Matematech envolve dispositivos eletrônicos de baixo custo e fácil acesso e não requer uso da Internet ou conhecimentos muito específicos dos docentes que vão utilizá-la. Outra meta alcançada está relacionada à efetividade da plataforma no auxílio ao aprendizado de matemática. A partir da análise de experimentos práticos realizados em uma escola com alunos do $4^{\circ}$ e $5^{\circ}$ ano, foi possível perceber o quão efetiva é a ajuda da plataforma para propiciar um conhecimento matemático mais contextualizado aos discentes dos anos iniciais do Ensino Fundamental. 
V Congresso Brasileiro de Informática na Educação (CBIE 2016)

Anais do XXVII Simpósio Brasileiro de Informática na Educação (SBIE 2016)

a trabalhos futuros, já se encontra em desenvolvimento uma versão da plataforma para computadores e outra para dispositivos móveis com sistema Android. Pretende-se integrar todos os formatos desenvolvidos por meio de uma base de dados única em que os usuários poderão participar de um ranking, motivando ainda mais o uso da solução proposta. Além disso, novos níveis de dificuldade serão implementados para que possam ser incluídos alunos dos anos finais do Ensino Fundamental.

\section{Referências}

AVVISATI, F. (2012). Programme for international student assessment (pisa) results from pisa 2012 - brazil. http://www.oecd.org/brazil/ PISA-2012-results-brazil.pdf. Acessado em fevereiro de 2016.

CARVALHO, M. A. G. (2005). Teoria dos grafos: uma introdução. Disponível em: http://www.ft.unicamp.br/ magic/ft024/apografoscesetmagic.pdf.

CARVALHO, R., ROSA, P., MACHADO, J. V., JÚNIOR, J. G. R. e COSTA, G. (2015). Ferramenta para auxílio na aprendizagem de lógica de programação em sistemas informatizados. I Workshop de Jogos Educativos Digitais Interdisciplinares (JEDI) 2015, p. 1144-1153.

EDUCAÇÃO, M. (2007). Plano de desenvolvimento da educação. Disponível em: http://www.mec.gov.br/pde. Acessado em março de 2016.

GONÇALVES, J. P. (2004). Reflexões sobre os processos de ensino/aprendizagem de matemática baseados no software educativo formel. Revista Brasileira de Informática na Educação, 12(2).

INEP (1998). Matrizes curriculares de referência para o saeb. Instituto Nacional de Estudos e Pesquisas Educacionais. Disponível em: htpp://portaldomec.com.br. Acessado em março de 2016.

NETO, S. R. S., SANTOS, W. O. e JUNIOR, C. G. S. (2013). Games no ensino da matemática: processos de virtualização de jogos para uso entre estudantes e professores da região agreste do estado de pernambuco. V Simpósio Hipertexto e tecnologias na educação, p. 216.

QEDU (2013). Aprendizado dos alunos: Brasil. Disponível em: http://www.qedu.org.br/brasil/aprendizado. Acessado em maio de 2016.

RIBEIRO, A. L. (2012). Jogos digitais online: ampliando o(s) letramento(s) na sala de aula. IV Simpósio Hipertexto e tecnologias na educação, p. 1-15.

TAVARES, W., helton Cristian de PAULA, e LIMA, M. A. e BARBOSA, F. V. (2012). Khan academy: Uma abordagem da escola construtivista ou o uso de novas ferramentas na abordagem da escola tradicional da educação. Novas Tecnologias na Educação, 10.

TODA, A. M., CARMOS, R. S. D., NETO, J. C., SILVA, A. L. e BRANCHER, J. D. (2014). Desenvolvimento de uma aplicação web para auxiliar no ensino da matemática para alunos do ensino fundamental. XXV Simpósio Brasileiro de Informática na Educação, p. 392-401.

VALLECILLOS, A. e MORENO, A. (2002). Framework for in-struction and assessment on elementary inferential statistics thinking. International Conference on the Teaching of Mathematics, 2nd(1):1-6. 